\section{PYORRHOEA ALVEOLARIS."}

\author{
BY
}

\author{
HUMPHREY HUMPHREYS, O.B.E., M.C., M.B., \\ CH.B., M.D.S., L.D.S. \\ HONORARY SURGFON, BIRMINGHAM DENTAL HOSPITAL.
}

THF ohject of the following remarks is to bring into focus such knowledge as we have on the subject of pyorrhoea, some important additions to which have been made in the last year or two. The inacculate and vague nature of its title is perhaps justified partly by the lack of certainty that shrouds its pathology, partly by its very general use, and I shall not devote time to a description of its signs and symptoms. I shall deal only with the condition most usually understood by " pyorrhoea alveolaris," the special features of which are its general distribution over the mouth and its extreme chronicity. This limitation will exclude from my purview, on the one hand, localized conditions such as the gingivitis, so often seen round the front teeth in mouth-breathers, and inflammation due to local injury, such as tooth restorations, dentures, and orthodontic treatment; and, on the other hand, the acute general conditions such as mar be caused by the chemical poisons mercury, phosphorus, and iodine, and special infections like those that produce nlcerative stomatitis, "trench gums," etc. We are left with a condition which, though the clinical picture varies from case to case, has as its lowest common denominator a progressive loss of the tooth-supporting structures; characterized by the two features already mentioned - general distribution and extreme chronicity.

\section{Pathology.}

Perhaps the most important fact in its pathology-which I think we may now safely regard as established-is a negative one: that is, that no specific organism plays the part of causa causans. It is now pretty generally accepted that the organisms present are those non-specific germs normally to be found in the mouth, generally streptococci and staphylococci, sometimes spirochaetes and fusiform bacilli. One qualification should perhaps be added. The formation of calculus is a constant and, as I shall show, a vitally important feature of the discase; and the work of Bulleid, since confirmed by other workers, shows that the Iicptothrix burculis plays an essential part in calculus formation, and may thus perhaps in a sense be regarded as a prime cause in the production of pyorrhoca. Our conception of the sequence of the pathological changes involved has been much clarified by the researches carried out in the last few years at the Bland-Sutton Institute of the Middlesex Hospital by Warwick James and Counsell, ${ }^{2}$ and by other workers, both in that institute and elsewhere. The most important points now made clear are:

(a) That the condition commences on the interdental septa and thence spreads both vertically into the periodontal memhrane and laterally on to the labiobuccal and lingual surfaces.

(b) That the initial lesion consists in a deep proliferation of the gingival epithelium at the expense both of the gum corium and of the periodontal membrane, and that pari passu with this deep proliferation goes a superficial desquamation, resulting after a time in the formation of a " pocket," which thus, throughout the whole course of the disease, continues to be lined with epithelium. (The discovery that normal healthy gum is attached to the enamel ${ }^{2}$ has compelled us to abandon the conception of a noimal "trough" between the gum and the crown of the tooth.)

(c) That calculus or "tartar" is invariably present, though not always in sufficient quantity to be detected macroscopically.

(d) That absorption of the alveolar bone and periodontal membrane is secondary to the primary epithelial change.

(e) That the discharge of pus from the pockets instead of being, as the name of the disease implies, an essential feature is to be regarded as a complication which, as we know from clinical experience, does not occur in a large proportion of cases. When it does occur it is due to a secondary infection of the pockets by pyogenic organisms with a positive chemiotaxis. Of course, when the pockets have become large a

${ }^{*}$ A post-graduatn Ierture delivered in Birmingham on November 8th, discharge of some sort can generally be expressed from them, but this is not normally pus, but the so-called materia alba, and consists chiefly of food debris and masses of putrefactive organisms.

\section{Causation.}

It is still considered by some that in pyorrhoea we have to deal not with a single disease, but with a group of diseases linked together by the fact that they all attack the same organ. This is suggested partly by the variation in the clinical picture, partly by the difference of the bacterial flora in different cases, and partly by analogy with other groups of imperfectly understood diseases, such as chronic rheumatism and rheumatoid arthritis. But just as physicians now appear to regard rheumatoid arthritis and chronic rheumatism more and more as due in nearly all cases to infection from some focus in the body, so I think we are now justified in believing pyorrhoea to be a single disease, and it is certainly a great aid to treatment if we can take up this positive standpoint. The differences we observe in the clinical picture must, then, be regarded as due to a difference in the reaction of the patient rather than to a difference in the cause. As in all other diseases, causes divide themselves into a predisposing. group and an exciting group. Most of the hoary controversies between the adrocates of a general or systemic and those of a local origin of pyorrhoea have had their root in the failure to appreciate that both a predisposing or general and an oxciting or local cause must always be present in every case.

\section{Predisposing Causes.}

The first of these is the essentially transient and temporary nature of the teeth and their supporting structures, a physiological fact due to the evolutionary history of the mammalia. 'The reptiles from which they sprang are polyphyodont-that is, they have a succession of teeth limited in numbers only by the life of the individual; each set after a short period of use is cast off and replaced by another. In that class of reptiles (the Theriodontia) from which it is bolieved that mammals were evolved we see that the life of each set of teeth became more and more prolonged, till in some species two sets were sufficient for the normal life of the individual. This arrangement became stereotyped in mammals, and all the sets of teeth after the second were suppressed. But throughout the mammalia, if an animal lives much beyond its normal allotted span, there is a tendency for its teeth to become lost, till it finally becomes edentulous. The exceptions are teeth of persistent growth, and if they are so arranged that no wear reduces their size these teeth continue to grow larger throughout life-for example, the tusks of elephants.

The loss of the permanent teeth due to wear and the atrophy of the tooth-supporting structures is, then, a normal feature of old age, and there is no clinical difference between the loss of tceth as a normal senile change and their jormature loss from pyorrhoea in middle life. Of the reasons for this prematurity in the victims of pyorrhoea we arc still ignorant. They may act either directly on the tooth-supporting structures, predisposing them to atrophy and absorption, or they may act by encouraging the deposit of calculus, the most vital of the exciting causes. They probably signify some biochemical change in lime metabolism, perhaps due to an altered endocrine activity. In this connexion F. W. Broderick ${ }^{3}$ and Weston Price ${ }^{4}$ have made some interesting suggestions, pointing out that pyorrhoea becomes-more common as caries becomes less common-a contrast that applies not only to the age incidence, but to the type of tooth most severely attackedand that pyorrhoea is a disease of lime excess in the blood, caries one of lime deficiency. But a coherent explanation of these matters has still to be worked out, and for the present we must be content to realize that there is undoubtedly some unknown predisposing cause which helps to determine why amongst individuals in whom the exciting causes are equally operative some are attacked by pyorrhoea and other's remain immune.

\section{Exciting Causes.}

While the predisposing causes are still obscure and are not as yet susceptible to treatment, the exciting causes are now beginning to be understood, and, what is more 
important, are to some extent capable of being controlled. They may be summed up in the single word "stagnation." Consider the pathological picture whose salicnt features I have described. The disease starts in the interdental spaces, the only position where even assiduous oral hygiene cannot entirely prevent stagnation from occurring. It is characterized by the deposit of calculus from the oral secretions, a deposit that can only occur when those secretions stagnate. It commences by a proliferation of epithelium, a process which all over the body denotes, except in the case of new growths (and often in this case also), a local irritant of some sort. The conclusion which obtrudes itself is that stagnation of the oral secretions in the interdental spaces-assisted, as we now believe, by the activities of the Leptothrix buccalis_produces a deposit of calculus; this, in its turn, acts as an irritant to the epithelium covering the interdental septum, causing it to proliferate deeply and desquamate superficially till a pocket is formed. A vicious circle is now established: the presence of the calculus and the existence of the pocket will still further encourage stagnation, not only of the cral secretions, but of fermentable food debris, and leads in turn to the formation of more calculus and deeper pockets, and the existence of a culture medium for bacterial growth. Infection from this, spreading along the blood vessels and lymphatics which pierce the crest of the bony interdental septa, produces absorption of the bone till every detail of the pathological picture is accounted for. If this conception covers the facts we have only to consider the factor's which favour oral stagnation.

1. The first and most important of these is modern dict. The teeth and jaws of man were evolved before cooking was invented, and the friction of a natural diet on the gums, with the thorough mastication involved by it, promotes a flow of the oral and salivary secretions sufficient to prevent any of that oral stagnation which is to some extent inevitable with modern food. The point is well illustrated by comparative pathology. Wild animals rarely if ever suffer from pyorrhoea apart from loss of the teeth in extreme old age. Animals, however, in captivity and domestic species are not infrequently attacked by the disease owing to their artificial diet.

2. Irregular arrangement of the tecth and malocclusion, due either to faulty development of the jaws or to premature loss of teeth, will obviously encourage stagnation, and wo find, in fact, that pyorrhoea is more common in such mouths, as it also is in mouths where the saliva is unduly viscid.

3. Dental restorations, whether fillings, crowns, bridges, or dentures which from faulty design or imperfect execution tend to trap food and impede a free flow of oral secretions, will obviously provide an exciting cause for pyorrhoea.

\section{Treatment.}

It is an axiom that all sound treatment of clisease should be directed towards its cause, and not to its symptoms. Over the predisposing causes of pyorrhoea we have as yet no control, and if the conception of its exciting causes which I have put before you is well founded it follows that our sole aim in treatment should be the continued prevention of oral stagnation; and in fact the conception of oral stagnation as its cause rests not only on the evidence of pathology, but on the clinical fact that the therapeutic measures directed against it are the only ones that do produce a permanent result. What measures, therefore, are open to us? We cannot expect to seo the adoption of a diet so coarse and astringent that it would be sufficient in itself, and the treatment possible divides itself into surgical and medical procedures.

\section{Surgical Treatment.}

The paramount surgical treatment is a complete removal of all the calculus, and this scaling, in the great majority of cases, will have to be repeated at regular intervals. It is obvious that, however thoroughly the calculus is removed, the cause that led to its deposit in the first instance will bring about the same condition again; and the medical measures I shall describe, directed to the removal of that cause, are seldom so thoroughly carried out or so perfectly effective that the need for subsequent scalings will not arise. Another surgical measure of great value is the abolition of any pockets that may exist-that is, the removal of the gum to the level of the floor of the pockets, by the knife, the cautery, or by escharotic drugs. The existence of pockets renders the task of preventing stagnation around the teeth, which is the goal of all our treatment, peculiarly difficult, and the clinical results of gum resection are uniformly excellent.

\section{Medical Treatment.}

The surgical measures I have mentioned, though they arrest the course of the disease for the time being, cannot be expected to effect a permanent cure. The patient will be no less liable to oral stagnation, a fresh deposit of calculus, and a recrudescence of the disease than he was before, and the surgical measures must be followed up by some daily measure of oral hygiene which will, as far as is humanly possible, prevent a recrudescence of pyorrhoe: by preventing oral stagnation. No measure that is not repeated daily can hope to maintain a permanent cure, and it follows that effective medical treatment can only bo undertaken by the patient, and will be effective in proportion to the diligence we can persuade him to practise.

There are three methods I have tried in my own practice: (1) daily irrigation of the gums and interdental spaces with a powerful spray from an atomizer; (2) daily cleansing of interdental spaces, and any pockets in the gum that are not resected, with hydrogen peroxide applied through a fine root syringe; and (3) saline massage. The salt promotes a flow of serum into the pockets and interdental spaces by osmosis; the massage first squeezes this out and then causes the pockets to fill up again with scrum. Stagnant areas, are thus sluiced out by the patient's own serum.

Daily medical treatment by the patient himself somewhat on these lines is, I believe, the only treatment likely to give results that are permanently satisfactory. The other forms of medical treatment are mostly aimed at what we now know to be complications of the disease, such as infection of the pockets with pyogenic organisms, rather than at the primary condition; and conditions such as pus formation usually disappear spontaneously in response to the measures I have mentioned. If they do not, then a course of special treatment may sometimes do good-cither the old method of treating the pockets with caustic drugs, or a course of vaccine therapy, or ionic medication, or treatment with high frequency current. Nothing is more easy than to effect a cure of pyorrhoea if by cure is meant an arrest of pus formation. Only too of ten the so-called cures have meant little more than success in eliminating this particular complication of the disease for the time being.

\section{Pyolrhoea and Systemic Infection.}

The time is not far distant when physicians were addicted to ordering the wholesale extraction of teeth attacked by pyorrhoea, in the interest of the patient's health. To-day the attitude of medical men appears to have changed, and we find their attention directed to teeth with non-vital pulps as the most important dental factor in general disease, together with an implicit reliance on $x$ rays as the only relíable method of diagnosis. Except that I believe radiographic evidence, taken alone, to be wholly inadequate and often misleading, I am fully in agreement with this attitude. The cases in my own clinical experience where teeth with pyorrhoea have damaged the general health are an insignificant minority compared with the vastly greater number where such damage has been wrought by non-vital teeth, and I find that physicians with sufficient knowledge of dental pathology clearly to distinguish between the two have the same experience. Weston Price, in the most extensive and important study of the dental factor in systemic disease yet published, ${ }^{4}$ goes even further and appears to regard active pyorrhoea as evidence of the establishment of a local quarantine or immunity reaçtion to the rheumatic group 
of diseases. Stanley Colyer has published a book ${ }^{5}$ taking the opposite view, and regards the alveolar bone in all cases of pyorrhoea, even where no pus is obvious, as infected. Unfortunately, in the cases he cites as evidence of improvement in health following extraction, he fails to distinguish between non-vital and vital teeth, and this seriously impairs their value. Lazarus-Barlow ${ }^{6}$ found that over 80 per cent. of cultures taken from the bone below the floor of a pyorrhoea pocket were sterile.

While it remains true that active pyorrhoea does not adversely affect the general health as much as was formerly believed, and far less frequently than apical infections, it is not to be thought that it is wholly innocuous. Its ill effects are most often seen in diseases of the digestive tract, as its toxic products drain into the mouth rather than into the blood; very few cases have occurred in my clinical practice where diseases of the rheumatic group were produced.

The relatively innocuous nature of pyorrhoea compared with apical infections is usually explained as the difference between an open and a closed infection, the toxic products of pyorrhoea draining freely into the mouth and the bacteria being killed by the gastric juices, while the toxic products of apical infections must of necessity find their way into the system. But there is another and more significant difference between the two conditions. An infection of the gums is freely exposed to all the defensive mechanisms of the body, phagocytes, antibodies, and antitoxins, which in most cases constitute an adequate defence. But an infected pulp chamber constitutes a stronghold for bacterial growth, completely protected from all the natural defence forces of the body, where germs can multiply and discharge their toxins and themselves into the system with impunity.

\section{References.}

1 A. Bulleid: Study of Leptothrix buccalis, Brit. Dental Journ., 1925, vol. xlvi, p. $289.2 \mathrm{~W}$. Warwick James and Counsell : Histological Investigation into so-called Pyorrhoea Alveolaris, ibill, 1927, vol. xlviii,

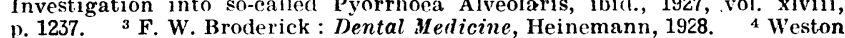
p. 1237. ${ }^{3} \mathrm{~F}$. W. Broderick : Dental Medicine, Heinemann, 1928. ${ }^{4}$ Weston
Price: Dental Infections, Oral and Systemic, Penton Publishing Co. Price: Dental Infections, Oral and Systemic, Penton Publishing Co.,
U.S.A., 1923. 5 Stanley Colver: Chronic Infection of the Jau's, Lewis U.S.A., 1923. ${ }^{5}$ Stanley Colver: Chronic Infection of the Jau's, Lewis
and Co. 6 . Lazarus-Rarlow : Bacteriological Examination of the and Co. ${ }^{6}$ P. Lazarus-Barlow: Bacteriological Examination of the
Alveolar Bone in Pyorrhoea, Brit. Dental Journ., 1928, vol. xlix, p. 57.

\section{NOTES ON A PEDIGREE OF AMAUROTIC FAMILY IDIOCY (TAY-SACHS DISEASE).}

BY

\section{D. STENHOUSE STEWART, M.R.C.S., L.R.C.P. D.O.M.S.}

HONORARY OPHTHALMIC SURGEON, VICTORIA HOSPITAL FOR SICK CHILDREN, HULL.

Intenest in this disease appears to have waned during the past twenty-eight years, and references to the condition are as few during this period as they are plentiful in the twenty years succeeding its first description by Waren Tay in 1881. The condition has apparently been shelved as incurable, largely because it seems to develop prenatally, and it has been impossible previously to forecast that the offspring of any particular Jewess was liable to this affliction.

It has been my good fortune to see at the Hull Victoria Hospital for Sick Children a case of this disease which has proved on investigation to represent the second generation of the classical R_ family. Their vicissitudes were recorded by Mr. Kingdon of Nottingham; of twelve children, six died of this complaint, and in two cases extensive post-mortem examinations were performed by Dr. Risien Russell.

Of the remaining six, the eldest daughter is unmarried; the elder son has one living child, one pregnancy terminated apparently in a "mole," and one other child died of meningitis secondary to mastoid trouble. $\mathbf{M y}$ patient was the second child of the next daughter. I do not intend to report the case fully, as it followed so typically the abler description of its uncles and aunt published by Mr. Kingdon in Volume 80 of the Transactions of the Medico-Chirurgical Society. Though flaccid throughout, the patient died convulsed. A boy born to the same mother seven years ago is alive and well.

The next son has married recently, shortly before he knew of the dangerous heredity, and he is disinclined to procreate on this account. The next living member of the family is a woman, who has a healthy daughter aged $2 \frac{1}{2}$ years, and is pregnant for the second time, expecting to be delivered in Apiril. The last is a woman of 21, and unmarried.

My object in publishing this pedigree is twofold. First, I consider that the appearance of the disease in the second generation is of general interest, and if it should occur elsewhere in the family it will be important to determine whether or no the succession is solely through the distaff line. Secondly, I desire the consensus of opinion of my colleagues on the manner they think best of meeting the situation. Should Jacob practise rigorous contraception? Should Rachel be advised against matrimony? Will the profession offer any suggestions as to the ante- and postnatal treatment of Pauline's expected child, and any future pregnancies of hers, Rebecca's, or Rachel's if she marries? Kingdon suggested that the dietary of the mother be investigated, and that the babies be weaned from birth, but that was at a time when the disease was regarded as familial rather than hereditary.
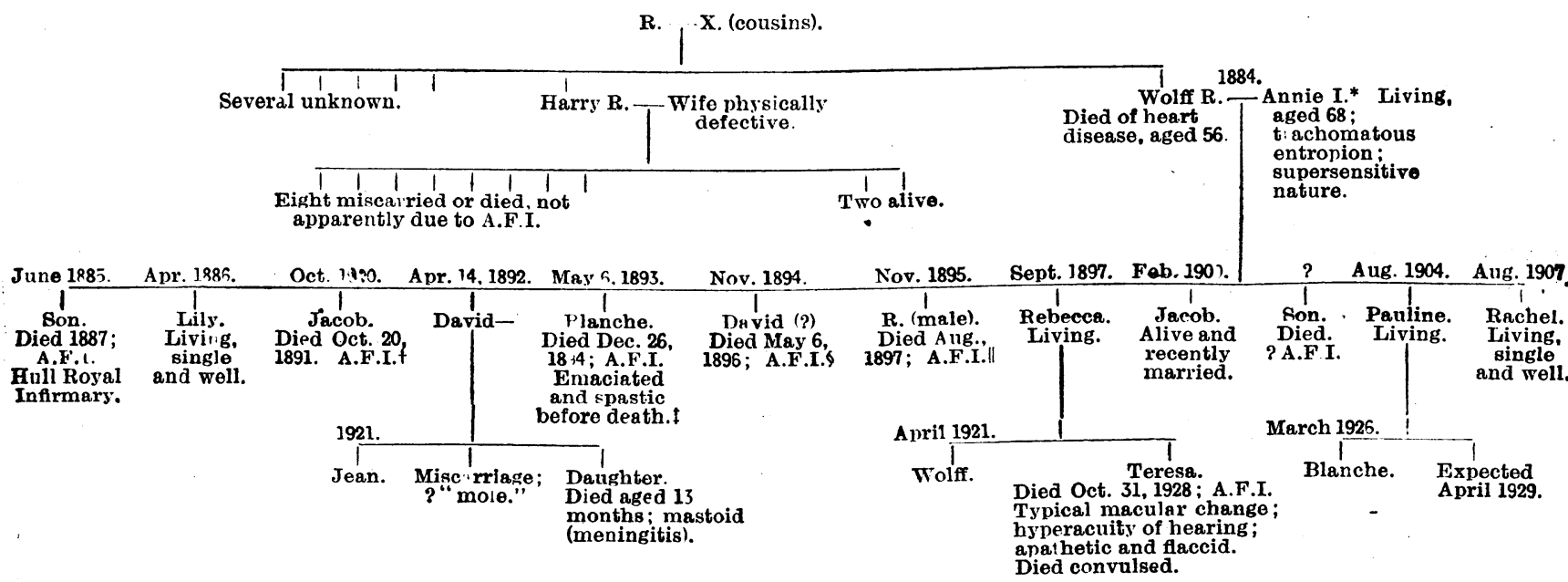

* Other nembers of this family have no abnormal history so far as lnown.

+ Tran actions of the Ophthalmological Societv, vol. xii, p. 126 Quoted in the T,ansactions of the Roval Medical and Chirurgical Society of London, $1 \times x \times, 87$ t Transactions of the Ophthalmological Society. Xiv, 129. Transactions of the Medico-Chirurgical Society, $1 \times x x, 87$. Chirurgical Society of London, II Ibid., Addendum. 